Recepción: 25/11/2018

Aceptación: 10/01/2019

Publicación: 05/03/2019

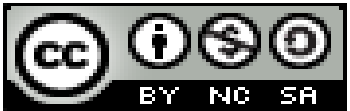

Ciencias económicas y empresariales

Artículo de investigación

\title{
Plan de negocios para la creación de una empresa procesadora y comercializadora de chocolate
}

Business plan for the creation of a chocolate processing and marketing company

\section{Plano de negócios para a criação de uma empresa de processamento e comercialização de chocolate}

\author{
Martha Alejandra Olivo-Olivo ${ }^{\mathrm{I}}$ \\ martha.olivo@ucacue.edu.ec \\ Hugo Jaime Avendaño-Fajardo II \\ hugo.avendano@ucacue.edu.ec
}

Correspondencia: martha.olivo@ucacue.edu.ec

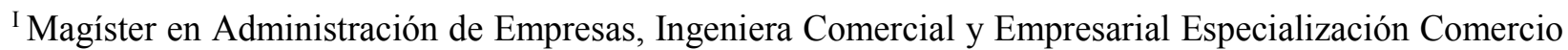
Exterior y Marketing, Docente Universidad Católica de Cuenca. Cuenca, Ecuador.

II Magíster en Contabilidad y Auditoría, Contador Público Autorizado, Docente Universidad Católica de Cuenca. Cuenca, Ecuador.
} 


\title{
Resumen
}

El presente artículo surge de un trabajo de maestría hecho por la autora, en el cual se planteó un estudio base cuyos objetivos fueron desarrollar un plan de negocios direccionado a la creación de una empresa procesadora y comercializadora de chocolate en el cantón el Triunfo de la provincia del Guayas, cuantificar la oferta y demanda del producto, establecer los aspectos técnicos, tecnológicos y administrativos del proyecto y determinar la factibilidad financiera con las proyecciones de ingreso y costos para el funcionamiento de la empresa procesadora de chocolate. El estudio hecho revelo una demanda insatisfecha en cuanto al objeto de estudio en dicho escenario. Se concluyó que es factible y rentable la creación de una empresa comercializadora y productora de chocolate, y se recomendó la aplicación, seguimiento y valoración del proyecto propuesto. Por ello, se ha querido expresar en este artículo lo que se ha considerado más relevante en cuanto a la temática desarrollada.

Palabras claves: Plan de negocios; procesadora y comercializadora de chocolate; empresas; factibilidad y rentabilidad.

\begin{abstract}
This article arises from a master's work done by the author, in which a basic study was proposed whose objectives were to develop a business plan aimed at the creation of a chocolate processing and marketing company in the county of El Triunfo in the province of Guayas, quantify the supply and demand of the product, establish the technical, technological and administrative aspects of the project and determine the financial feasibility with the projections of income and costs for the operation of the chocolate processing company. The study revealed an unsatisfied demand regarding the object of study in this scenario. It was concluded that the creation of a chocolate marketing and production company is feasible and profitable, and the application, monitoring and evaluation of the proposed project was recommended. Therefore, we wanted to express in this article what has been considered most relevant in terms of the theme developed
\end{abstract}

Keywords: Business plan; chocolate processor and marketer; companies; feasibility and profitability. 


\section{Resumo}

Este artigo surge de um trabalho de mestrado feito pelo autor, no qual foi proposto um estudo básico cujos objetivos eram desenvolver um plano de negócios visando a criação de uma empresa de processamento e comercialização de chocolate no município de El Triunfo, na província. Guayas, quantificar a oferta e a demanda do produto, estabelecer os aspectos técnicos, tecnológicos e administrativos do projeto e determinar a viabilidade financeira com as projeções de receitas e custos para a operação da empresa de processamento de chocolate. $\mathrm{O}$ estudo revelou uma demanda insatisfeita em relação ao objeto de estudo no referido cenário. Concluiu-se que a criação de uma empresa de comercialização e produção de chocolates é factível e rentável, sendo recomendada a aplicação, acompanhamento e avaliação do projeto proposto. Portanto, queríamos expressar neste artigo o que foi considerado mais relevante em termos do tema desenvolvido

Keywords: Plano de negócios; transformação e comercialização de chocolate; empresas; Viabilidade e rentabilidade.

\section{Introducción}

El chocolate en el Ecuador en los últimos años ha experimentado mayor favoritismo en el consumidor interno, debido a lo cual el plan de negocios para la creación de una empresa procesadora y comercializadora de chocolate en el cantón El Triunfo provincia del Guayas se tomó en consideración para el mercado-meta.

Del proceso de recolección de información generado mediante la aplicación de encuestas a las tiendas de los mercados potenciales (Cantón El Triunfo, Cantón Duran, y Norte de la ciudad de Guayaquil) se pudo establecer que el $82 \%$ de la muestra son consumidores de chocolate, por otra parte de esta demanda estimada solo se llega a satisfacer el $38 \%$ de la misma, en la mencionada localidad, principalmente se planea satisfacer a las tiendas ofreciéndoles un producto de buena calidad y precios que sean accesible al proveedor. Mediante encuesta realizada a los dueños de las tiendas se pudo evidenciar que el $80,21 \%$ está de acuerdo que compraría otra marca de chocolate que le permita obtener mayor utilidad sobre sus ventas.

En lo que respecta al mercado global el cacao y sus derivados representan una amplia demanda de consumo, generando amplias tendencias a nivel nacional en lo que respecta a procesos de exportación, favoreciendo ampliamente los niveles de producción y venta de este producto, 
posibilitando un movimiento económico fundamental para el Ecuador. Partiendo de los aspectos previamente mencionados es posible considerar que el desarrollo de un plan de negocios orientado a la creación de una empresa procesadora y comercializadores de chocolate en el cantón El Triunfo, Provincia del Guayas, se traduciría en un compendio de información tanto teórica como practica que beneficiaría al sector industrial del Ecuador y de la Provincia; convirtiéndose además en el punto de partida para el mejoramiento técnico de los procesos de producción y comercialización, en lo que respecta a la industria.

La investigación realizada al respecto, se orientó al estudio de los procesos de producción y comercialización del chocolate, producto seleccionado por la amplia demanda que ocupa dentro del mercado, acción llevada a cabo mediante la generación de un plan de negocios que encuentra conformado por diferentes etapas de estudio, permitiendo así la determinación de aspectos específicos, como lo es el nivel de la oferta y la demanda del producto, los aspectos técnicos organizacionales necesarios, así como los legales y los niveles de inversión que permitirán el desarrollo de un emprendimiento de esta magnitud.

A pesar de ser un mercado que puede generar un amplio rendimiento económico a largo plazo además de ser uno de los mercados que representan una amplia demanda, en el Ecuador hay escaza presencia de empresas procesadoras y productoras de chocolate por falta de financiamiento debido al costo de materia y especialmente de la maquinaria que hace posible la producción generando un amplio costo de elaboración del producto terminado.

Actualmente no se han generado nuevos emprendimientos orientados al proceso del cacao y la comercialización del chocolate, fundamentalmente se puede notar que una empresa de estas características, propicia el mejoramiento de la utilidad para los productores al incrementarse paulatinamente el consumo, de la misma manera actúa en beneficio de los obreros que ocuparan las plazas de trabajo que se originaran tras su implementación posterior funcionamiento.

Es preciso tomar en cuenta que a pesar de que el Ecuador es un país productor de chocolate, aun es necesario mejorar y potencializar adecuadamente su producción, puesto que la misma no cubre la demanda existente, pese a que la promoción del consumo del chocolate nacional es muy pobre, es decir, si hubiera una mayor promoción del chocolate nacional, la demanda podría

\section{6}

Pol. Con. (Edición núm. 31) Vol. 4, No 3, marzo 2019, pp. 103-118, ISSN: 2550 - 682X 
potencializarse abriendo una gran oportunidad a la producción de chocolate, lo que es necesario para el mercado interno, puesto que la materia prima nacional como tal es más aprovechada por los exportadores, lo que no deja iguales ganancias.

El desarrollo de la investigación se justificó en primera instancia, por la importancia que representa el sector agrícola para el dinamismo de la economía ecuatoriana, así como el sector manufacturero de cacao y sus derivados; la elaboración de un plan de negocios orientado a la producción y comercialización de este producto, permitió la determinación de aspectos técnicos operacionales y niveles de inversión necesarios para este tipo de emprendimientos.

La factibilidad de la investigación se dio debido a la facilidad de recolección de información de carácter teórica proveniente directamente de textos especializados, así como la información de campo proveniente de la aplicación de instrumentos de información como los son las encuestas.

La importancia de la investigación se dio, debido a que por medio del desarrollo del plan de negocios fue posible determinar los procesos necesarios para la generación de este tipo de emprendimiento, dando a conocer los niveles de la oferta y la demanda dentro del cantón El Triunfo; siendo además un compendio de información que permitirá el mejoramiento de los procesos de producción y comercialización

Otro factor que en muchos casos representa una desventaja es la falta de tecnología diseñada para el procesamiento de la materia prima; sin embargo existe la imperiosa necesidad de superar este obstáculo mediante la creación de procesos tecnificado que posibiliten una ventaja estratégica en el tiempo y proceso de producción, desarrollando cadenas de producción de alto rendimiento y de muy buena calidad; con estas bases se puede desarrollar un protocolo que permita la capacitación del personal en el manejo de procesos altamente tecnificados y adquirir este tipo de tecnología y hacer uso de ella en la producción de chocolate de alta calidad y buena rentabilidad.

Además, el no desarrollar estudios adecuados que permitan determinar un mercado óptimo para este producto, propicia que se sigan implementando procesos tradicionales y artesanales al no tener una óptica adecuada del negocio, es así que como consecuencia hay un pobre mejoramiento de calidad y de industrialización del producto terminado, que es el chocolate. 
En base a esta problemática este trabajo pretende ser un aporte al sector agrícola, especialmente cacaotero mediante la elaboración y análisis del plan de negocios para desarrollar una empresa productora y procesadora de chocolate que implemente nuevos procesos, aplicación de tecnología y el desarrollo de constante control y mejoramiento genético para la obtención de chocolate, siendo así, se plantea el cuestionamiento:¿Es posible la elaboración de un plan de negocios con su debido análisis orientado al desarrollo de una empresa productora y comercializadora de chocolate en el cantón El Triunfo en la Provincia de Guayas?

En concreto se quiere desarrollar un plan de negocios direccionado a la creación de una empresa procesadora y comercializadora de chocolate en el cantón el Triunfo de la provincia del Guayas Para ello se cuantifica la oferta y demanda del producto en los mercados potenciales de El Triunfo, Durán y Guayaquil, estableciendo los aspectos técnicos, tecnológicos y administrativos del proyecto, a fin de determinar la factibilidad financiera con las proyecciones de ingreso y costos para el funcionamiento de la empresa procesadora de chocolate.

\section{El sustento teórico}

Desde el aspecto teórico investigación hecha, se planteó como finalidad esencial, el diagnosticar la situación acerca del desarrollo empresarial, del mercado de la producción y proceso del cacao en el cantón El Triunfo provincia del Guayas, mismo que beneficia al desarrollo del sector agrícola puesto que aportara con nuevas fuentes de ingreso que permitirán el desarrollo empresarial.

Sobre las generalidades del Cacao es importante destacar como lo plantea Guayas, (2013) que, cacao es el nombre científico del árbol del cacao o cacaotero, de la familia Malvaceae. Theobroma significa en griego alimento de los dioses; cacao deriva del nahua cacáhua.

Corona, (2014) conceptualiza que el árbol de cacao es una planta tropical de copa densa, hojas perennes de color verde brillante, flores de color blanco rosado, directamente insertadas sobre el tronco o las ramas viejas y fruto grande en forma de baya ovoide, de color rojo marrón; puede sobrepasar los 8 metros de altura y señala que la elaboración del chocolate procede del procesamiento del grano de cacao. A lo largo de la historia el chocolate ha tenido connotación

\section{8}

Pol. Con. (Edición núm. 31) Vol. 4, No 3, marzo 2019, pp. 103-118, ISSN: 2550 - 682X 
social, religiosa, medicinal, política y económica en diversas sociedades en la actualidad su propagación es tal que en los actuales momentos no existe región donde no se consuma.

Al respecto Quevedo, (2013), informa que alrededor de mundo su consumo y producción involucra a millones de personas y naciones, inicialmente el chocolate fue consumido por las tribus Olmecas de Mesoamérica, hace tres mil años como bebida y después empleado como moneda por los Mayas, después de la conquista de México, este llego a ser conocido en Europa, principalmente en España donde se cambia la fórmula inicial, se le agrega azúcar de caña y aromatiza con canela, logrando inicialmente una gran aceptación social

En la intención de este artículo es importante ahora señalar que, se denomina Plan de Negocios a la estructura programática tanto técnica, financiera y económica, de todos los factores que deben ser tomados en cuenta, tanto para la implementación como parta la puesta en funcionamiento de un nuevo emprendimiento o de la ampliación del negocio, esto con la finalidad de identificar su vialidad y su rentabilidad de forma previa. Un plan de negocios es una estructura organizada de varios procesos fundamentales para que sea factible la realización del proyecto planteado, siendo así consta de: Estudio de Mercado, Estudio Técnico, Plan de Marketing, Estudio Organizacional, y Estudio Financiero.

Del plan de negocios depende el éxito de la empresa, este deberá contener todo lo necesario para el adecuado funcionamiento de la empresa se consolida con elemento de mucha importancia que permiten conocer el mercado, así como los procesos requeridos para la producción consideran inclusive la maquinaria que deberá proveerse para llegar a la elaboración de producto, también se sintetizan todos la promoción requerida, la organización incluyendo lo correspondiente al ámbito legal, así como también los rubros a ser invertidos en el nuevo emprendimiento.

En cuanto a lo que se refiere a estudio de mercado, podemos señalar que para Ferre (2010) un estudio de mercado es un proceso sistemático de recolección y análisis de datos e información acerca de los clientes, competidores y el mercado. El uso que se le dé a esta información incluye ayudar a crear un plan de negocios, lanzar un nuevo producto o servicio, mejorar productos o servicios y expandirse a nuevos mercados.

Por lo tanto al realizar un estudio de mercado antes de iniciar cualquier negocio por muy pequeño que este sea, es importante porque favorece la selección de estrategias y acciones a la 
implementación de un nuevo negocio, de tal manera que se obtenga la rentabilidad deseada a los inversionistas, haciéndolo autosustentable a futuro, el conocimiento del mercado es el que brinda las pautas para identificar si el giro del negocio es bueno, como se comporta el consumidor, la competencia, el nivel de demanda existente en el mercado, en que área se podrá distribuir el producto con mayores niveles de venta que garanticen un mayor éxito a la compañía, principalmente en los primeros años que corresponden con el periodo de posicionamiento de la marca.

Esto es necesario completarlo con conceptos sobre el análisis de la oferta y la demanda. Según lo que indica Pino, (2006) la oferta y la demanda no se presentan en forma independiente, si no que se dan al mismo tiempo, porque en el mercado hay alguien que desea vender un producto y otro que desea comprar el mismo producto a un determinado precio. Estas son dos variables de mucha importancia que se pueden medir o determinar aproximadamente por medio del estudio de mercado, la oferta es el número de productos existentes en el mercado destinados a la satisfacer las unidades requeridas por los clientes consumidores.

Mientras que por su parte la demanda es el número de clientes existentes, así como la frecuencia con la que los clientes existentes, están en deseo y condición de adquirir un producto. El análisis de la demanda es uno de los paseos iniciales y sin duda uno de los de mayor importancia, el poder identificar el volumen de cliente que tiene el producto en un área determinada, para medir las posibilidades de que el producto de la nueva empresa participe de una buena cantidad de clientes (Benalcazar, 2010).

En cuanto al análisis de la oferta, Esquivel (2006) manifiesta que la oferta es el conjunto de bienes y servicios que se ofrecen en el mercado en un momento determinado y con un precio accesible. Para el estudio que estamos refiriendo, la población se determinó en 13641 tiendas entre los cantones de El Triunfo, Guayaquil y Duran considerando el censo económico del INEC para el periodo correspondiente al año 2012, por tanto el mercado objetivo del actual análisis, se determina en el 75,94\%; lo que representa en 10.359 de la demanda insatisfecha en número de tiendas que se detalla mediante el anexo $\mathrm{N}^{\circ} 8$ este dato es de suma importancia puesto que es preciso proveer a la empresa de la logística necesaria para la atención de las tiendas.

\section{0}

Pol. Con. (Edición núm. 31) Vol. 4, No 3, marzo 2019, pp. 103-118, ISSN: 2550 - 682X 
Existen empresas tanto nacionales como internacionales que tienen una importante participación en el mercado, principalmente en lo que respecta a las tiendas de abarrotes que se constituyen en el mercado meta a ser alcanzado por "Inlate SA" entre ellas las de mayor presencia en este segmento del mercado son: Nestle, Bono Bon, Jet, Noggy, La Universal, Ferrero, Nicolo y Anuta.

Según Merchan (2012). es una gran ventaja el poder contar con un mercado lleno de una diversidad de marcas, puesto que esto da a entender que los clientes permiten la participación de nuevas marcas y naturalmente las acogen sin dificultad En el Ecuador existen diversas marcas que elaboran el producto y lo distribuyen, tales como: La Universal, Ferrero, Nestlé y entre otras que ya han sido mencionadas en el punto anterior; estas por lo general llegan a través de mayoristas y distribuidores a las tiendas grandes y pequeñas. La gran mayoría de estas empresas gozan de ser compañías bien estructuradas, su capital es de naturaleza privada, es decir, este es un mercado muy competitivo en relación a la calidad de los productos (Bedoya, 2011).

Según Zambrano (2014). estas empresas cuentan con buenos procesos productivos de alta calidad y con certificación INEN y correspondiente registro sanitario, impulsan muchas plazas de empleo directo e indirecto, estas empresas cuentan con publicidad en medios escritos, en radio y televisión, así como un gran uso de la publicidad exterior, situación que se realiza obviamente para posicionar la marca en la mentalidad del cliente y ganar su preferencia, frente a las demás Estudio Técnico.

\section{Metodología}

Debido a las características de la investigación, se optó por una modalidad cuali-cuantitativa cuyo proceso se enfoca en la recolección de información de carácter medible y cuantificable (Bunge, 2004), por medio de este fue posible la determinación de los niveles de la oferta y la demanda del mercado, sirviendo además como respaldo para el desarrollo del análisis de las inversiones a ser necesarias para el funcionamiento de la empresa.

El tipo de investigación fue de carácter no experimental, proceso que se orienta para el análisis de las variables en su entorno directo de desarrollo, es decir, que por medio de este tipo de investigación se analizaron los niveles de oferta y demanda del mercado, frecuencia de compra y 
el volumen de producción que se presentan actualmente en la comercialización de chocolate en el mercado nacional, mediante estos conocimientos se sustenta una propuesta de viable factibilidad.

La investigación se basó en dos universos, la primera con la finalidad de determinar los niveles de compra y venta del producto dentro del cantón El Triunfo así como de dos cantones cercanos que son Guayaquil y Duran, en los cuales se pretende tener una considerable incidencia, por ello se selección como primer universo las principales tiendas que se encuentran en dichas localidades, dando un total de 13.641 se les aplicara formula de muestra para determinar la aplicación de los instrumentos de recolección de información.

\section{Resultados}

Al consultar de sobre el producto más vendido en los locales comerciales obtuvimos que un $52,67 \%$ tienen preferencia por el chocolate negro, mientras que el 30,75\% manifiestan inclinación por el chocolate con algún ingrediente adicional (maní, almendras, arroz crocante), por su parte el 9,89\% disfrutan más del chocolate en polvo (cocoa, chocolisto, etc), un 4,55\% de la población encuestada comercializa aún más la presentación en bombones, finalmente el 2,14\% vende con mayor frecuencia el chocolate fino importado.

Se pudo establecer que el $54,81 \%$ de las tiendas encuestadas adquieren el producto de los mayoristas, es decir, de centros comerciales que compran mayor volumen y lo comercializan a menor precio, la diferencia del precio adquirido con la del precio de venta al público se constituye en la ganancia de este tipo de tienda; mientras que el 45,19\% de las tiendas encuestadas adquieren el producto en las distribuidoras, que son empresas que adquieren el producto directamente de los productores a menor precio para distribuir a las tiendas, siempre dejando un margen de ganancia para los tienderos; quedando el 0,00\% para las fábricas.

Cuando se consultó sobre la marca más vendida nos encontramos que 70,32\% de los productos que se expenden en las tiendas consultadas son marcas nacionales ya posicionadas como la universal, mientras que el $29,68 \%$ son marcas internacionales

El chocolate es un producto de gran demanda y aceptación en el público, el estudio de mercado revelo que existe una demanda del $82 \%$ y en la actualidad solo se llega al 38\% de los clientes; lo que representa una gran oportunidad para ubicar el producto en el mercado, haciendo énfasis en

\section{2}

Pol. Con. (Edición núm. 31) Vol. 4, No 3, marzo 2019, pp. 103-118, ISSN: 2550 - 682X 
las tiendas, despensas, mini Market, supermercados que serán los principales clientes de la empresa Inlate SA. Por lo tanto, se estima que en el cantón El Triunfo, Guayaquil y Duran; la empresa tiene grandes oportunidades de crecer y brindar beneficios a la economía de la localidad, mediante la creación de nuevas y estables plazas de empleo, así como produciendo productos de muy alta calidad; siendo el consumo anual per cápita de chocolate en Ecuador oscila entre los 300 y 800 gramos por persona al año, mientras que en Alemania promedia los nueve kilos. Esa cifra revela que aún falta impulsar el consumo local en el Ecuador, según analistas y productores consultados (Revita Lideres, 2015).

La oferta actual cubre un $38 \%$ del mercado aproximadamente según el estudio de mercado realizado, esta oferta está compuesta tanto por empresas nacionales como por empresas internacionales; siendo las segundas las predominantes como es el caso de Nestlé SA, Ferrero SA, entre otras; mientras que la nacional de mayor presencia en el mercado es La Universal SA. En Ecuador operan 143 establecimientos donde se vende chocolate y confitería que facturaron en su ejercicio 2009 más de \$ 786 millones según el Censo Nacional Económico (INEC, 2010).

\section{Discusión}

Lo planteado es el desarrollo de un plan de negocios para una empresa destinada a procesar y comercializar chocolate en el cantón El Triunfo y la provincia del Guayas; así como en la realización de los estudios de mercado, técnico, plan de marketing, organizacional y financiero. Estos propósitos han sido muy coherentes y han permitido el desarrollo de una investigación muy completa que servirá de base para inversionistas que deseen incursionar en empresas de este tipo, teniendo en cuenta es que Ecuador goza de ser reconocido como un país productor de cacao de óptima calidad, por ende, de un excelente chocolate, ventaja que puede ser aprovechada para emprender en la producción de chocolate.

El plan de negocios realizado se constituye en una guía completa para la creación de la empresa Inlate SA, posibilitando así un importante aporte teórico como práctico que permite el Cuantificar la oferta y demanda del producto en los mercados potenciales de El Triunfo, Durán y Guayaquil.

De acuerdo con el autor Fernández, (2012) en su libro "investigación y técnicas de mercado" se define que, en una sociedad capitalista, los precios no se determinan por una autoridad central, sino que son el resultado de cómo los compradores y vendedores interactúan en los mercados. Un 
mercado, en un sentido económico, no es más que una colección de compradores y vendedores de un producto o servicio en particular. Sin embargo, a diferencia de un mercado físico, compradores y los vendedores no tienen que estar todos en el mismo lugar, sino que sólo tienen que tener la capacidad de llevar a cabo la misma transacción económica. Ahí es donde entra en juego el modelo de la oferta y la demanda. Desde el siglo XIX, la oferta y la demanda ha sido un factor determinante en el sistema capitalista.

Dada la importancia que representa la demanda en lo que corresponde a los mercados y la fijación de precios de los productos, se realizó el debido estudio de mercado utilizando principalmente la aplicación de las encuestas donde se pudo determinar la demanda insatisfecha del producto objeto del presente estudio, dentro del cual se estima que, expresado en número de unidades esta sería de 17’515.794 anual, representada por un promedio de población a atender de 13.641 .

En lo que respecta a la oferta referente a la competencia existente en el mercado se determina que la producción y comercialización del chocolate se encuentra distribuida entre 13 empresas y su proporción de participación es Nestle SA 28\%, Confiteca CA 22\%, Ferrero del Ecuador 17\%, Ecuacocoa 7\%, Adams del Ecuador 6\%, otras marcas 6\%, Ecuador Cocoa y coffee Ecuacoofee SA con un 5\%, Cacaos finos Ecuatorinos 3\%, Pérez Bermeo 2\%, Erolcorp SA 1\%, Gustaff SA $1 \%$, Comsaju CIA Ltda. $1 \%$ y finalmente Confitecor $1 \%$.

En base a la competencia y la aceptación de los posibles consumidores se determinó que la oferta de la empresa será de 5’254.738 de unidades anual que equivalen al 30\% de la demanda total insatisfecha, y se encuentra representada por un promedio de población a atender de 10.359 personas.

Con el antecedente expuesto se establece que en lo que corresponde al tamaño del proyecto, este tendrá capacidad de producir 5’400.000 productos derivados de chocolate procesado; de igual forma la capacidad será de producir $22.500 \mathrm{Kg}$. De derivados de cacao diarios; y la ubicación más favorable es el cantón El Triunfo por tener bajos costos en servicio básicos y en la zona se encuentran los proveedores de la materia prima minimizando los costos de adquisición de la 
misma, además por su cercanía con los mercados de distribución del producto el costo de transporte para posibilitar el consumo del mismo es mínimo.

Mediante el respectivo estudio organizacional se determinó la distribución administrativa y el personal necesario para un correcto funcionamiento de la empresa, objeto del presente estudio; de igual forma el perfil necesario para cada cargo a ser ocupado entre personal administrativo y operativo.

Determinar la factibilidad financiera con las proyecciones de ingreso y costos para el funcionamiento de la empresa procesadora de chocolate.

Lo planteado es el desarrollo de un plan de negocios para una empresa destinada a procesar y comercializar chocolate en el cantón El Triunfo y la provincia del Guayas; así como en la realización de los estudios de mercado, técnico, plan de marketing, organizacional y financiero.

Estos propósitos han sido muy coherentes y han permitido el desarrollo de una investigación muy completa que servirá de base para inversionistas que deseen incursionar en empresas de este tipo, teniendo en cuenta es que Ecuador goza de ser reconocido como un país productor de cacao de óptima calidad, por ende, de un excelente chocolate, ventaja que puede ser aprovechada para emprender en la producción de chocolate.

El plan de negocios realizado se constituye en una guía completa para la creación de la empresa Inlate SA, posibilitando así un importante aporte teórico como práctico.

\section{Conclusiones}

Existe una demanda de 8'736.408 unidades de chocolate de 100gr faltantes los que se constituyen en la oportunidad para participar en el mercado del chocolate en los cantones en los que se realizó la investigación y porque no a nivel nacional. Según el estudio realizado la oferta existente cubre un $38 \%$ de la demanda y en su mayoría es realizada por empresas extranjeras como es el caso de Nestlé SA, Ferrero SA, entre otras.

La empresa tendrá la capacidad de producir $22500 \mathrm{~kg}$ de chocolate al día, sin embargo no se planea iniciar al máximo de la capacidad, sino ir incrementándola a medida que se vaya insertando el producto al mercado. 
La planta de Inlate SA se ubicará en el cantón El Triunfo con el fin de disminuir movimientos migratorios de fuerza laboral en este cantón y poder brindar una oportunidad de crecimiento económico a esta población.

La planta contara con dos bloques bien definidos uno direccionado al proceso de transformación de la materia prima (producción) y otro dedicado a la dirección de la empresa (administrativo).

El proceso productivo consta de ocho pasos que son: recepción de la materia prima, despulpadora, mezclado con azúcar, refinado, hidratación, llenado de moldes, envoltura y control de calidad.

La empresa será una sociedad anónima, regida por la Junta General de Accionistas y representada por un/a gerente. Es altamente factible económicamente, se pretenden la venta de aproximadamente $940 \mathrm{TN}$ de chocolate anualmente.

\section{Recomendaciones}

Aprovechar que existe una demanda insatisfecha para participar del mercado del chocolate, por ello es importante que se realice lo antes posible a fin de que se gane clientela de forma ágil.

Es imprescindible la creación de empresas netamente ecuatorianas que procesen el chocolate con la finalidad de incremental la participación del valor agregado ecuatoriano en este mercado.

Se debe tener en cuenta que a planta tenga una buena capacidad de producción con la finalidad de que, al presentarse una mayor acogida a los productos de la empresa, esta pueda ser aprovechad.

Dentro de lo posible se recomienda la contratación de mano de obra local con el fin de potenciar el desarrollo económico de la localidad.

Los bloques que componen la empresa deben trabajar de forma coordinada a fin de conseguir una buena dirección y producción puesto que la segunda depende de la primera y viceversa.

Es fundamental que el procedimiento de producción en cada paso se considere un alto control de calidad y sanidad, esto con la finalidad de garantizar productos de excelencia al mercado. 
La empresa deberá responder responsablemente, ante cada uno de los organismos gubernamentales como son la Superintendencia de Compañías y el SRI, así como su gerente, esto incrementara la confianza de los clientes, así como de los socios y de los posibles inversionistas.

Se debe incrementar continuamente las metas de venta del chocolate en la empresa, trabajando siempre para alcanzar la visión institución y lograr una importante participación en el mercado.

\section{Referencias Bibliográficas}

Andrade, M. (2007). Chocolate en el Ecuador y en el mundo . Manabí: Samary.

Bedoya, H. (2011). Desarrollo de la empresa privada y publica. Caracas: Bittec.

Bunge, M. (2004). La investigación cientifica: su estrategia y su filosofía. Mexico DF: Siglo XXI editores SA.

Corona, B. (2014). Generalidades del cacao. Ecuador: Norma.

Fernandez, J. (2012). produccion y la forma de potencializar los mercados . Bogota: ENNA.

Ferre Trenzano, J. (2010). El estudi de mercado. MADRID: DIAZ DE SANTOS S.A.

Guayas, L. P. (22 de Julio de 2013). municipiodeltriunfo.gob.ec. Obtenido de http://www.guayas.gob.ec/cantones/el-triunfo

INEC. (2010). Censo de población y vivienda. Quito : Instituto Nacional de Estadisticas y Censos.

INEC. (2010). Censo Nacional Economico. Ecuador: Instituto Nacional de Estadisticas y Censos.

Martinez, A. (15 de 08 de 2016). www.metroecuador.com.ec. Obtenido de www.metroecuador.com.ec: http://www.metroecuador.com.ec/noticias/el-cacao-nacionales-bien-recibido-por-el-consumidor-extranjero/rUroez---AVNUdyDCiX6s/

Merchan, C. (2012). La selva de los negocios. Santiago de Chile : La estrella.

Molina, A. (2012). Oportunidades a la vista. Quito: Icaza.

Parales, D. (2006). Estudio Tecnico. Madrid: SOS. 
Pino, R. (2006). Correspondenccia entre oferta y demanda. Caracas: Chaco.

PRO ECUADOR. (2015). Perfil sectorial de cacao y elaborados para el inversionista. Quito: Instyituto de Promocion de Exportaciones e Inversiones.

Quevedo, R. (2013). El cacao. Mexico: Espin.

Revita Lideres. (12 de 08 de 2015). Revista Lideres. Obtenido de Revista Lideres: http://www.revistalideres.ec/lideres/sabor-dulce-preferido-ecuador

Zambrano, J. (2014). Implicaciones de la Publicidad en el mercado. Madrid: Goreti. 